\title{
On suitability of the reinforcement learning methodology in dynamic, heterogeneous, self-optimizing networks
}

\author{
Milos Rovcanin, Eli De Poorter, Ingrid Moerman, Piet Demeester \\ Ghent University - iMinds, Department of Information Technology (INTEC) \\ Gaston Crommenlaan 8, Bus 201, 9050 Ghent, Belgium \\ Email: milos.rovcanin@intec.ugent.be
}

\begin{abstract}
An ever growing number of deployed wireless networks dictates a tempo with which the inter-network cooperation techniques are being developed. Cooperation, in this sense, can go far beyond a simple activation of an interference avoidance techniques. This paper describes and evaluates the performance of a reinforcement learning based reasoning engine, used in a selflearning, cognitively controlled cooperation between heterogeneous, co-located networks. Coupled with a concept of cooperation through the network service negotiation, this approach represents an efficient, yet scalable solution for the dynamic network selfoptimization.
\end{abstract}

Index Terms-Self-learning, reinforcement learning, network optimization, network service negotiation;

\section{INTRODUCTION}

There is a growing need for network solutions that dynamically support at run-time cooperation between devices from different networks [1]. Currently, the only way to support connectivity between co-located devices is to statically group them into different sub-nets, depending on their communication technology. This approach ensures that the same network policies are used for a sub-net, regardless of the characteristics of the devices. However, the process is usually quite complex and it might lead to a huge waste of available resources.

Dynamic, at run-time management is expected to improve many networking aspects [2]: decrease energy consumption, decrease interference, improve availability and bandwidth allocation etc. The major issue here is the fact that different sub-nets usually have different requirements (high level goals), that must not be neglected. Otherwise, this can lead to a misbehavior some of the participating sub-nets and, once again, an unacceptable waste of available resources.

Obviously, there is a need for an intelligent entity that controls and supervises the process of establishing an internetwork cooperation. Having it performed through a process of activating or deactivating network services [3], this reasoning entity will have to be able to determine the optimal set of services for each one of the participating sub-nets. An additional dimension to a problem is given by the fact that activation of a service in one sub-net, consequently influences the performance of all the involved sub-nets.

Reasoning engine, proposed here, is based on an on-line, reinforcement learning methodology that does not require any sort of an a priori knowledge about the influences that different services pose on the network high level goals. Instead, it uses a number of network features to make the precise assessment of them. Collected features are used as an input for the Least Squares Temporal Difference (LSTDQ) [5] algorithm that generates numerical values for the system performance at each state. The highest value is given to the best performing service set, which is therefore considered to be the optimal one, considering the given high level network goals.

Performance is continuously evaluated using the most recent data. No deprecated values are used, as it was the case with many of the previous machine learning approaches. By constantly collecting measurements from the environment, our engine is capable of noticing sudden condition changes and adapting to them.

The following chapters of the paper are organized as follows: Section III describes the fundamentals of the LSTDQ algorithm and its strong points, in regards to other machine learning approaches. Section IV discusses the suitability and prospects of using the LSTDQ in our use case. The following Section $\mathrm{V}$ gives a detailed overview of the experimentation set up, used for the proof of concept and testing. Section VI presents results and discussion regarding each one of them. Section VII proposes possible improvements and future course of development. Finally, Section VIII summarizes and concludes the paper.

\section{RELATED WORK}

Work presented here is considered to be an improvement of an already used paradigm [3], which describes inter-network cooperation through an activation of network services in colocated networks. The process is initiated and controlled by a linear programming based reasoning entity - CPLEX ILPSolver [4], but requires design-time knowledge of the impact of a network service on the network performance.

\section{LEAST SQUARES TEMPORAL DifFERENCE ALGORITHM - LSTDQ}

\section{A. Fundamentals}

When modeled using the reinforcement learning approach [6] [7], a problem transforms into a Markov Decision Process (MDP), with the continuous or discrete set of states $S=s_{1}, s_{2}, s_{3}, \ldots, s_{n}$. A decision maker passes from one state 
to another by selecting an action at every step. Set of actions can also be continuous or discrete, $A=a_{1}, a_{2}, a_{3}, \ldots, a_{n}$. A reward is given upon taking each action. Learning, in this case, considers using actions, thus following the decision making paths that maximize rewards:

$$
Q(s, a)=r(s, a)+\gamma \sum_{s^{\prime}} P\left(s^{\prime} \mid s, a\right) \max Q\left(s^{\prime}, a^{\prime}\right)(1)
$$

Above given is the well known Bellman equation, where $Q(s, a)$ represents the state-action function, also known as the $\mathrm{Q}$ function. It assigns numeric value to every state-action pair from the problem's state-action-space, based on the immediate reward $r(s, a)$, given for taking an action $a$ at the state $s$ and the future expected reward $\sum_{s^{\prime}} P\left(s^{\prime} \mid s, a\right) \max Q\left(s^{\prime}, a^{\prime}\right)$. The last argument includes the state-action transition probability, which is generally not known a priory.

\section{B. Approximations and $L S T D Q$}

LSTDQ was first introduced by M.G.Lagoudakis and R.Parr in [5] and further elaborated in [8], as a part of the well known Least Squares Policy Algorithm (LSPI). The algorithm is founded on the idea of representing the $\mathrm{Q}$ function as a linear combination of a certain number of problem features, basis functions:

$$
Q(s, a ; w)=\sum_{k} \phi_{j}(s, a) \omega_{j}
$$

Argument $\omega_{j}$ is the weight parameter. Basis functions are arbitrary and generally non-linear functions of $s$ and $a$. Their linear independence ensures no redundancy. Generally, the number of basis functions $k$ is significantly smaller than the number $|S||A|$ of state/action pairs.

Combining equations (1) and (2) yields a system:

$$
\begin{gathered}
\omega=A^{-1} b \\
\text { where: } A=\Phi^{T}\left(\Phi-\gamma P^{\pi} \Phi\right) \\
b=\Phi^{T} R
\end{gathered}
$$

Theoretically, in order to populate matrices $A$ and $b$, one must acquire information regarding basis functions and rewards for each pair from the problem's state-action space, which is not practical in large problem spaces and impossible if the problem space is continuous. Therefore, approximations are used.

Samples $D=\left(s_{d_{i}}, a_{d_{i}}, s_{d_{i}}^{\prime}, r_{d_{i}} \mid i=1,2, \ldots, L\right)$ are collected. Here, $\left(s_{d_{i}}, a_{d_{i}}\right)$ is sampled from the state-action space and $\left(s_{d_{i}}^{\prime}\right)$ is sampled according to $P\left(s_{d_{i}}^{\prime} \mid s_{d_{i}}, a_{d_{i}}\right)$ that is defined by the used sampling policy (a random policy uniform distribution). Argument $L$ represents the number of gathered samples. Finally, here are the approximated versions of the above given matrices:

$$
\begin{gathered}
\hat{\Phi}=\left(\begin{array}{c}
\phi\left(s_{1}, a_{1}\right)^{T} \\
\cdots \\
\phi\left(s_{n}, a_{n}\right)^{T}
\end{array}\right) \widehat{P^{\pi} \Phi}=\left(\begin{array}{c}
\phi\left(s_{1}^{\prime}, \pi\left(s^{\prime}\right)\right)^{T} \\
\cdots \\
\phi\left(s_{n}^{\prime}, \pi\left(s^{\prime}\right)\right)^{T}
\end{array}\right) \\
\hat{R}=\left(\begin{array}{c}
r_{1} \\
\cdots \\
\mathrm{r}_{2}
\end{array}\right)
\end{gathered}
$$

Matrices $A$ and $b$ can now be approximated in the following way:

$$
\hat{A}=\hat{\Phi^{T}}\left(\hat{\Phi}-\gamma P^{\hat{\pi}} \Phi\right) \text { and } \hat{b}=\hat{\Phi^{T}} \hat{R}
$$

Originally, LSPI was introduced as an off-line learning technique that starts with an initial policy and iterates it, over the same training set of training samples, in order to converge to its optimal outlook. Every iteration considers using the LSTDQ to calculate the respective weight vector so that two iteration can be compared. An on-line version was introduced later, in []. In this form, the LSPI does not use an acquired training set to iterate on policies, but continuously acquires samples and adds them (updates) the already existing set to correct weight factors and yield better $\mathrm{Q}$ value approximations. The common part of both approaches is the underlying LSTDQ engine that uses whatever set of samples is given to it to calculate the corresponding weight vector.

Implementation complexity and processing power needed for the LSTDQ depends on the number of samples it has to deal with. Dealing with smaller number of samples will reduce complexity, but depending on the size of the entire state-action space, it might have an unacceptable precision. The consistency between approximated and true values $A$ and $b$ matrices are given by these claims:

$$
E(\hat{A})=\frac{L}{|S||A|} A \text { and } E(\hat{b})=\frac{L}{|S||A|} b
$$

The following section will explain how the same LSTDQ based approach can be used for the purpose of inter-network self-optimization.

\section{LSTDQ - SUITABILITY AND PROSPECTS}

At the beginning, being a core of the LSPI algorithm, LSTDQ was primarily used in an offline type of approach. An off-line method demands a training set of samples to be formed prior to the learning process. During each policy iteration, samples are picked from the training set and fed to the LSTDQ algorithm. The corresponding set of weights is calculated.

Within an on-line approach, samples are gathered during the learning process. In the extreme case, weights are recalculated after each new sample is collected. Obviously, an on-line mechanism does not iterate policies in a number of distinct trials, but continuously, sample by sample. Cross comparison between an on-line and off-line LSPI algorithms is elaborated in details and published in [9].

We utilize an on-line method, without defining stopping rules. Consequently, no optimal policy is proclaimed. This makes sense since we are dealing with a dynamic environment. Policy determined as the optimal one might yield sub-optimal results after the change a network properties occur. Therefore, continuous sample collection is performed for two reasons:

- Fine tuning of the weight parameters $W=$ $w_{1}, w_{2}, w_{3}, \ldots, w_{k}$

- Detection of a network condition change 
Markov decision process, in our case, is memoryless. In other words, performance of the network at the current state does not depend on previous states it has been through. This property is then used to update up to $N_{\text {states }} \mathrm{Q}$ values after each learning episode. In use cases with relatively small number of states, this leads to an extremely fast collection of all the samples from the state-action space.

LSTDQ is invoked for the first time once all the samples are collected. It calculates the vector of weight factors for this sample set and determines $\mathrm{Q}$ values for every state-action pair. The following samples are collected using the " $\epsilon$ greedy" exploration technique [10]. At each state, with $\epsilon$ probability, our reasoning engine picks the action with the highest $\mathrm{Q}$ value. With $1-\epsilon$ probability, the action is picked at random. LSTDQ gets invoked every time a new sample is obtained.

Obviously, the $\epsilon$ factor (exploration factor) has the major influence on the algorithm. It directly influences both the time that system spends in the optimal/sub-optimal states and engine's capability to quickly respond to the change of network conditions. Further elaboration through experimental results and discussion will be given in the following sections.

\section{LSTDQ HowTo}

\section{A. Use case and experimentation set up}

We consider a case of two co-located wireless sensor networks. In the reminder of the paper, they will be referred to as network A and network B (see Figure 1). Process of establishing a communication between networks A and B [3] is out of the scope of this paper. It is assumed that networks $\mathrm{A}$ and $\mathrm{B}$ are already able of communicating with each other.

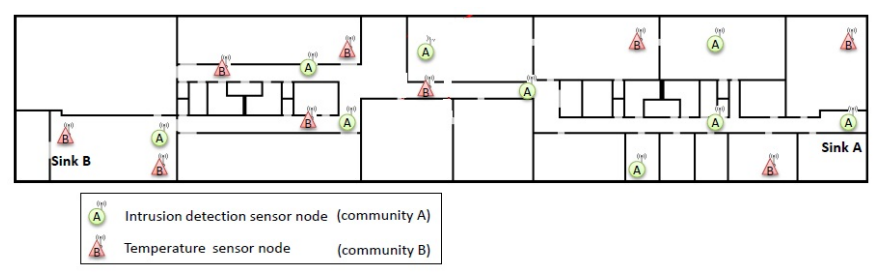

Fig. 1. Experimentation network topology. Part of the WiLab.t testbed [11], located at the iMinds research facilities, University of Ghent, Belgium

At this early stage of research, live measurements are still unavailable. Therefore, we used fabricated, artificial measurements, affected by the influence of fictive network services. Network A provides a set of fictive services $N_{S 1}=$ Service A, Service B. Similarly, network B provides services $N_{S 2}=$ Service C , Service D. Given the high level goals of network A: High Network Lifetime and Low Average Delay (both are assigned the same priority), the prime objective of the cognitive engine is to determine the optimal set of services in both networks so that the performance of network A, regarding its high level goals, is maximized.

\section{B. Implementation details}

For this use case, the state is determined by the joint combination of active/non-active services in both networks. Given the set of four services $N_{S 1}$ and $N_{S 2}$, the total number of states is $N_{\text {states }}=16$. The states are: $S_{t}=$ $A, B, C, D, A B, A C, A D, B C, \ldots, A B C D$. Performance of the network in each state is determined following the general rules set-up bu the system designer:

- Service A - always improves performance in a fixed manner

- Service B - improves performance when activated alone, degrades when combined with C,D,E

- Service C - improves performance when combined with $\mathrm{D}$, degrades when combined with $\mathrm{A}$ and $\mathrm{B}$

- Service D - best performing state when combined with $\mathrm{C}$ and $\mathrm{A}$, minor improvement/degradation with others

Combined in this manner, state $\mathrm{CB}$ represents the worst possible service combination, while DCA is the optimal set of services.

To be able to calculate $\mathrm{Q}$ values for each state-action pair, two basis functions are used: $\phi_{1}$ - end-to-end delay and $\phi_{2}$ - average number of re-transmissions. As stated before, measurements regarding basis functions are fabricated, not obtained during the run-time. $\mathrm{Q}$ values are calculated in the following manner:

$$
Q(s, a)=\phi_{1} \omega_{1}+\phi_{2} \omega_{2}
$$

Cognitive engine is allowed to switch between any of the two states, which means that the set of 16 actions $\left(A_{c}=\right.$ $\left.a_{1}, a_{2}, a_{3}, \ldots, a_{16}\right)$ is available at each state. The engine is ultimately expected to determine which state is the optimal one and force that respective service combination. Forcing a certain service combination in this context mean forcing transitions from any given state to the optimal one. If the system is already in the in the optimal state, the optimal decision would be to remain in it.

\section{Exhaustive exploration phase}

To take an advantage of the memoryless property of our set up, the first 16 episodes are used for an exhaustive exploration over the entire state-action space. All the possible states are investigated using a pseudo-random walk. Upon completion of this phase, LSTDQ is invoked using the gathered samples as an input data. The initial set of weight factors $W=\omega_{1}, \omega_{2}$ is calculated, followed by the calculation of the $Q$ values for every state-action pair.

Exhaustive exploration will make an initial differentiation between "good" and "bad" states. Furthermore, it will pinpoint the best and the worst performing states. However, forcing this initial policy from then on will make our cognitive engine rigid and not capable of adapting to a dynamic environment behavior.

\section{Exploitation phase}

Exploitation of the collected data begins after all the necessary samples are collected - upon completion of the exhaustive 
exploration phase. The process is conducted in the " $\epsilon$ greedy" fashion (Section IV). An appropriate values of the $\epsilon$ factor allow the reasoning engine to enforce the optimal set of services as much as possible, while still "being fair", up to an acceptable level, to sub-optimal states. Frequency with which the engine checks the sub-optimal states strongly affects its capability of noticing environmental disturbances. On the other hand, the system should not be kept "too long" in the states where it's performance is sub-optimal. Certain trade-offs are inevitable.

\section{RESULTS AND DISCUSSIONS}

Experimental results are presented in three separate subsections:

- Behavior of the reasoning engine in a static environment

- Behavior of the reasoning engine after a condition disturbance

- Behavior after utilizing a simple optimization technique

Explanation about the obtained results are given at the end of each subsection.

\section{A. Static environment conditions}

Figure 2 depicts initial Q values, calculated per each state upon completion of the algorithm's exploration phase. Since values of our basis functions depend exclusively of the destination state( ??), Q values for transferring from any state to a designated one are equal.

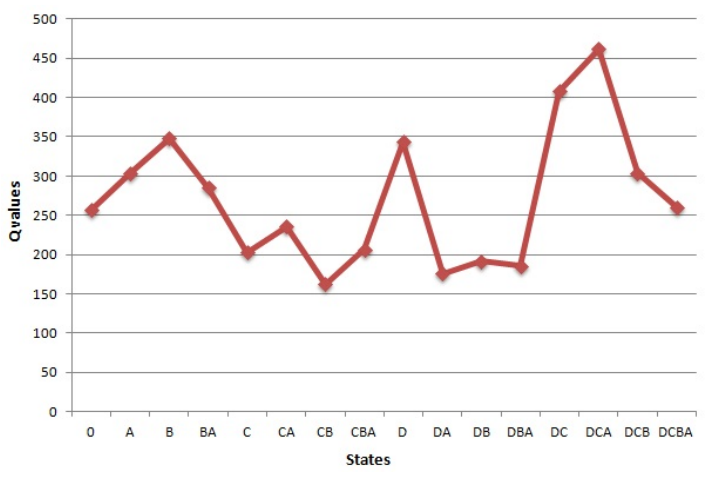

Fig. 2. Defined system states and their corresponding $Q$ values upon completion of the exploration phase

The best performing state, DCA, considers Service A, Service B and Service C to be activated, while Service B should be kept inactive. Exploitation phase, initiated at the end of the exploration phase, relays on these $\mathrm{Q}$ values .

The following Figure 3 depicts percentages of the number of learning episodes the system spent in each state during 100 learning episodes of the exploitation phase. Results are sorted in respect to values of the $\epsilon$ parameter.

As expected, the best performing state is determined to be CBA - services A and B activated in the Network A and service C activated in the Network B. Service D should be kept inactive. Corresponding percentages clearly depict the dependence on the value of the $\epsilon$ factor. After the exploration

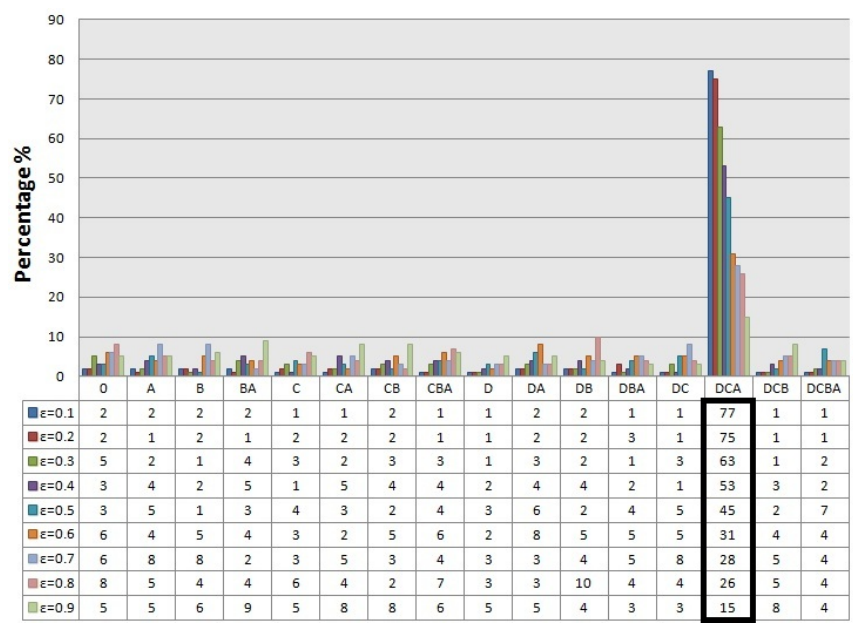

Fig. 3. Percentages ( $\mathrm{Y}$ - axis) are given in respect to the corresponding values of the $\epsilon$ factor, for every defined system state (X - axis).

phase is over and the initial $Q$ values are calculated, instances of the algorithm with the lower $\epsilon$ values tend to keep the system in the optimal state for as much as possible. The percentages vary from 77 down to only 15 percent, in cases when $\epsilon$ was set to 0.1 and 0.9 , respectively. It is worth noticing that, for the $\epsilon$ values of 0.4 and lower, our cognitive engine keeps the system in the optimal state for more than 50

During the exploitation phase, $\omega$ factor are being constantly recalculated and $q$ values reshaped. The following Figure 4 illustrate this process in three distinct cases, with the $\epsilon$ values set to $0.1,0.5$ and 0.9 , respectively:
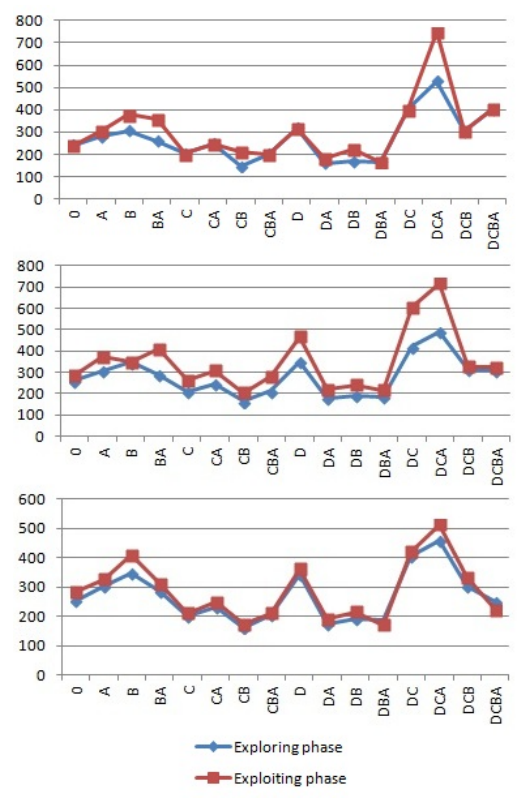

Fig. 4. Behavior of the $\mathrm{Q}$ values during the exploitation phase, in regards to different values of the $\epsilon$ factor. Values are set to $0.1,0,4$ and 0.9 , respectively

Algorithm instances (exploration policies) with lower $\epsilon$ values shape the $\mathrm{Q}$ values so that the optimal one is being 
increased at the highest rate. It appears as if the the suboptimal ones are being repressed. This is an expected behavior since every visit to a certain state shapes the $\omega$ factors in its favor, thus constantly increasing the corresponding $Q$ values. As opposed, in the case with an almost completely random policy $(\epsilon=0.9)$ the states are picked almost uniformly, without favorizing any in particular. Therefore, $Q$ values remain shaped similarly as after the exploration phase.

\section{B. Reaction to a network condition disturbance}

Exploitation phase has two closely related objectives:

- Updating the sample set after each learning episode, thus reshaping the $\omega$ factors so that the respective $Q$ values can be updated.

- Detects network condition disturbances and reshapes the decision making policy accordingly

The main indicator that the conditions in the network have changed are the $\mathrm{Q}$ values, calculated using re-shaped weight factors and respective basis functions. The following Figure 5 depicts the number of episodes needed to notice a condition change in the "worst case" scenario - best and worst performing states switch places (DCA - CB).

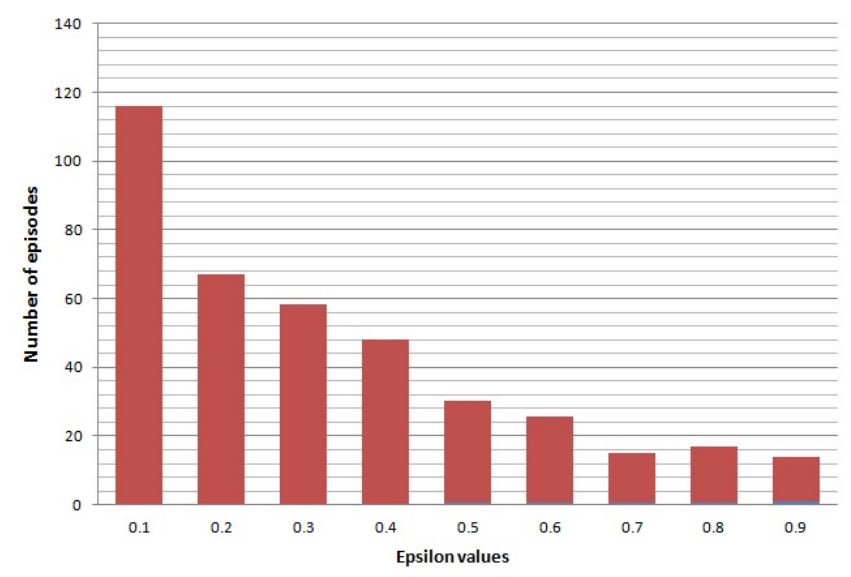

Fig. 5. Number of episodes needed to notice a network disturbance. The results are given in respect to different values of the $\epsilon$ exploration factor

The results in Figure 5 are averaged over 10 trials of 250 learning episodes. As expected, the quickest response is achieved with $\epsilon=0.9$ (13.2 episodes), since this mechanism checks system states at near-uniform manner. In the worst case, when $\epsilon=0.1$, it takes almost 120 episodes, in average, to react on disturbances and re-shape $\mathrm{Q}$ values accordingly.

Results presented in sections VI-B and VI-A reveal one major issue of the taken approach - how to find a compromising solution for the $\epsilon$ value, so that the system is kept in the optimal (or the nearest-to-optimal states) for as long as possible, while being able to "quickly" react to environmental changes? For an illustration, with $\epsilon=0.5$, system is kept in the optimal state for 45 percent of time, while engines ability to notice condition changes was limited to around 30 episodes, on average.
Fixing the $\epsilon$ to a certain value throughout the entire exploitation phase obviously does not provide acceptable results neither from the condition change versatility nor from the optimality point of view. One simple improvement of algorithm's efficiency during the exploitation phase is described and evaluated in the following sub-section.

\section{A simple efficiency-improving procedure}

In environments where the worst case scenario is impossible or rarely expected (moderate network dynamics), it is safe to restrain the exploitation phase to an optimal and a number of near-optimal states. This methodology can be applied in certain use cases where the traffic intensity does not change drastically over time. Figure 6 depicts the case where all the states with below the 50 percent of the optimal performance are discarded after the exploration phase.

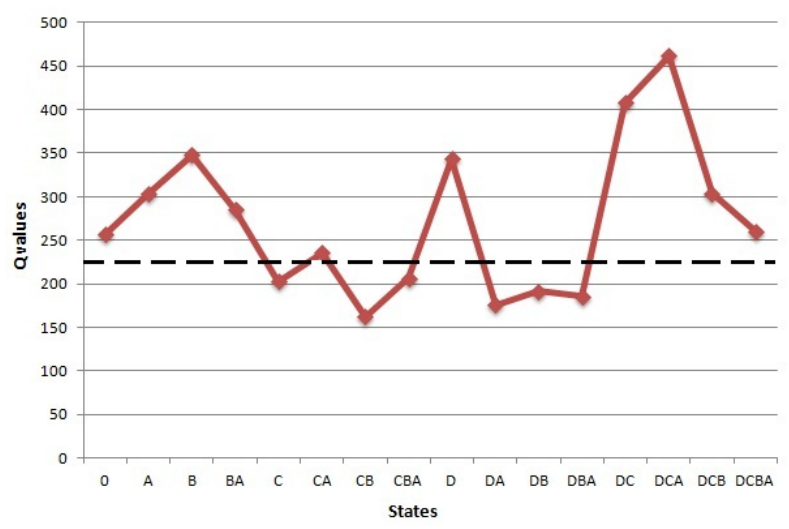

Fig. 6. Applying a threshold on $\mathrm{Q}$ values, prior to initiation of the exploitation phase. Threshold is set to 50 percent of the highest $\mathrm{Q}$ value

In this case, an applied threshold will rule out six worst performing states - C, CB, CBA, DA, DB, DBA. The reduced set of ten remaining states is taken into account during the exploitation phase. The following Figure 7 describes the percentage of the number of episodes that are spent in each state during 100 episodes long exploitation phase, given different values of the $\epsilon$ factor:

Interestingly, there is no significant difference in the number of episodes spent in the optimal state for almost all the values of $\epsilon$. However, by applying a threshold, the number of episodes spent in the worst performing states is significantly reduced. More importantly, the number of episodes needed to detect condition changes is reduced, as depicted on Figure 8:

Except for the case when $\epsilon=0.1$ a noticeable improvement is recorded during each run. Using a fixed value, $\epsilon=0.5$, system is kept in the optimal state 44 percent of time, with an average condition change response time of around 15 episodes. If we take into account that an additional 33 percent of time is spent in nearest-to-optimal states (DC, D, B), as opposed to 12 percent when no threshold is applied. Conclusively, even such a simple speed up procedure yields a considerable performance improvement. 


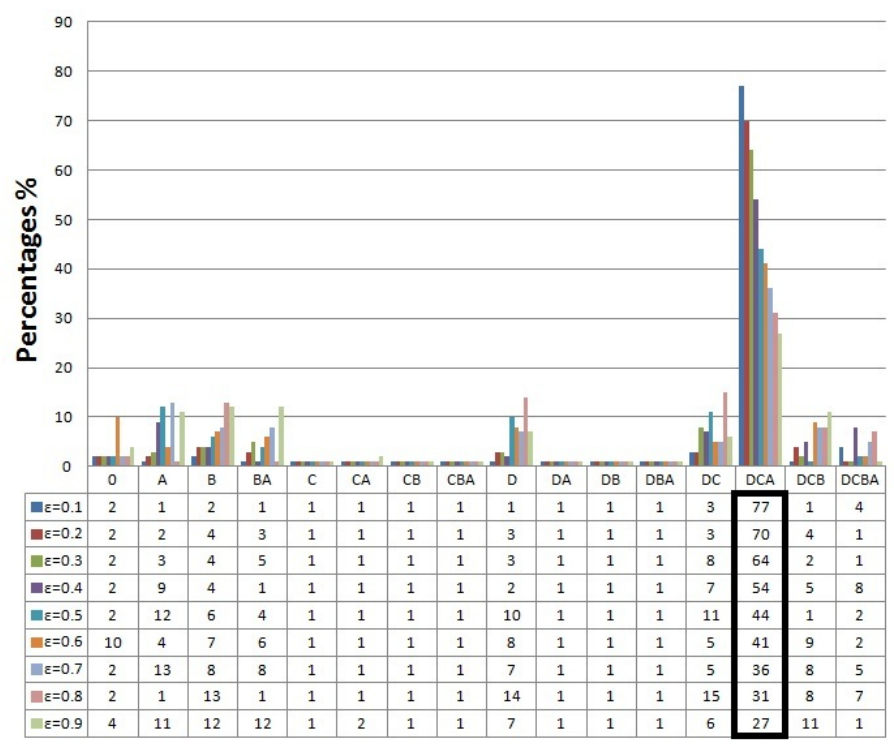

Fig. 7. Percentages ( $\mathrm{Y}$ - axis) are given in respect to the corresponding values of the $\epsilon$ factor, for every defined system state (X - axis), upon applying a simple efficiency-improving procedure

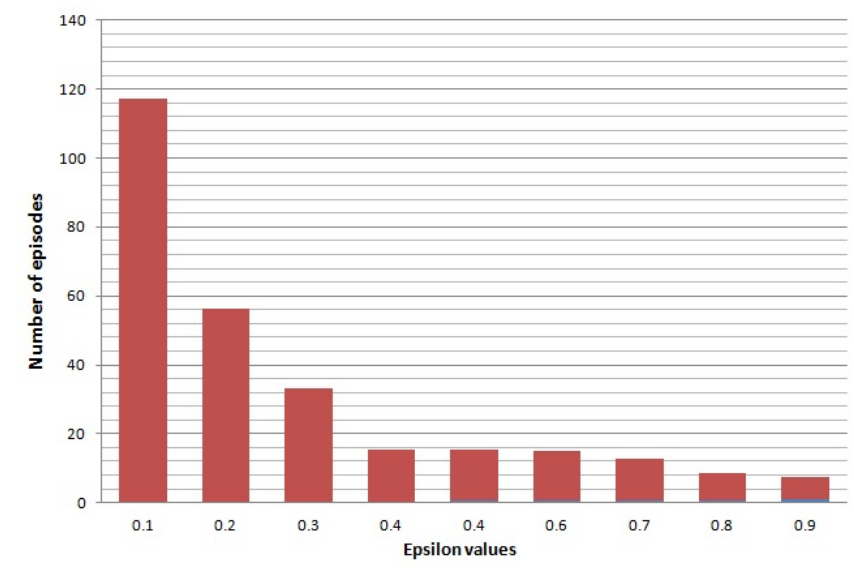

Fig. 8. Number of episodes needed to notice a network disturbance, while applying a simple speed up rule. The results are given in respect to different values of the $\epsilon$ exploration factor

More advanced techniques will be presented as the part of the future work, in the VII section.

\section{FUTURE WORK}

Future work implies improving the existing approach as well as expanding it on additional use cases. An at most attention needs to be paid on increasing the algorithm's efficiency during the exploitation phase, since efficiency is expected to become the major issue in use cases with the large state-action spaces.

One initiative is to develop techniques to "intelligently" reduce the number of system states that are being investigated. An already mentioned "performance threshold" approach is the simplest one. Others may also involve interpolation or prediction of a state performance. Both techniques aim at describing the performance of a system in states which have not been visited before. Some level of a priori knowledge can help defining a number of possibly forbidden states (e.g. certain services cannot be active at the same time). These techniques will have the major impact on efficiency once the duration of a learning episode becomes a factor.

Additional methods should focus on a dynamical change of the $\epsilon$ value during run-time. A critical network parameter, such as traffic intensity, is a good indicator of the network's overall behavior. Abrupt changes can be used to trigger an increase of the $\epsilon$ factor, thus stimulating an inspection of suboptimal states. Depending on a use case, it might be reasonable to introduce a certain probability distribution over available actions, according to their respective $\mathrm{Q}$ values, according to which actions will be taken when the " $\epsilon$ greedy" is utilized.

An additional use cases this approach can be expanded to are:

- Optimizing a performance of a single network protocol

- Optimizing a negotiation process between multiple networks and coordinating the cooperation

The main goal of optimizing a single protocol would be to determine the optimal set of protocol settings. Setting combinations will represent system states. Therefore, complex services, with a large number of settings that need to be tweaked will imply a large number of state-action pairs. The above mentioned techniques for increasing the algorithm's efficiency will be reused here as well.

To optimize multiple co-existing networks, some compromises will be inevitable. Metrics that will precisely describe whether certain compromise is justified or not, from each subnet's and the entire network's point of view, will have to be designed.

\section{CONCLUSION}

Optimizing multiple co-located networks, each with a variable number of network functionalities, influencing each other, is a complex problem that has not yet received a substantial attention in the research community.

This paper proposes and evaluates an application of the LSTDQ based, reinforcement learning approach. Our use case aims at discovering the optimal operating point of a single network, participating in a symbiotic cooperation with colocated, in general case, heterogeneous networks. Influences of the neighboring sub-nets are taken into account when calculating the optimal operational point. However, as opposed to most of the other approaches, no a priori knowledge about the influences is needed.

Use case described in this paper represents the proof of concept. Both strong and weak points of the implementation have been pointed out, along with a number of possible solutions and efficiency-improving techniques, all part of the future work. Most importantly, the final result of the learning process will be used as a starting point in the process of internetwork cooperation negotiation. Applying the same algorithm to all co-located sub-nets will yield an optimal, as well as the number of near-optimal operational points. They will represent a solid foundation in the following negotiation process. 
We strongly believe that the problem of interfering colocated networks will only increase. As such, innovative crosslayer and cross-network solutions that take these interactions into account will be of a great importance to the successful development of efficient next-generation networks in heterogeneous environments.

\section{ACKNOWLEDGMENT}

This research is funded by the Institute for the Promotion of Innovation through Science and Technology in Flanders(IWTVlaanderen)through the IWT SymbioNets project, by iMinds through the QoCON project and by the FWO-Flanders through a FWO post-doctoral research grant for Eli De Poorter

\section{REFERENCES}

[1] Wakamiya, N.; Arakawa, S.; Murata, M.,"Self-Organization Based Network Architecture for New Generation Networks", 2009 First International Conference on Emerging Network Intelligence, pp.61-68, 11-16 Oct. 2009

[2] R. W. Thomas, L. A. DaSilva and A. B. MacKenzie, "Cognitive networks", Proc. IEEE DySPAN 2005, pp.352-60

[3] E. De Poorter, B. Latre, I. Moerman and P. Demeester, "Symbiotic networks: Towards a new level of cooperation between wireless networks", Published in Special Issue of the Wireless Personal Communications Journal, Springer Netherlands, 45(4):479-495, June 2008

[4] http://www-01.ibm.com/software/commerce/optimization/linearprogramming

[5] M. Lagoudakis and R. Parr. "Model-free least-squares policy iteration". In Proc. of NIPS, 2001.

[6] T. G. Dietterich, and O. Langley, (2007) "Machine Learning for Cognitive Networks:Technology Assessment and Research Challenges in Cognitive Networks: Towards Self Aware Networks", John Wiley and Sons, Ltd, Chichester,UK. doi: 10.1002/9780470515143.ch5

[7] L. P. Kaelblign, M. L. Littman, A. W.Moore, "Reinforcement learning: A Survey", Journal of Artificial Intelligence Research 4 (1996) 237-285

[8] Michail G. Lagoudakis and Ronald Parr, "Least-Squares Policy Iteration, Journal of Machine Learning Research, 4, 2003, pp. 1107-1149.

[9] Lucian Busoniu, Robert Babuska, Bart De Schutter, Damien Ernst, "Reinforcement Learning and Dynamic Programming Using Function Approximators", 2010 by Taylor and Francis Group, LLC, ISBN 9781-4398-2108-4 (Hardback)

[10] John Myles White, "Bandit Algorithms for Website Optimization", Published by O'Rilley Media, Inc.

[11] Lieven Tytgat, Bart Jooris, Pieter De Mil, Benot Latr, Ingrid Moerman and Piet Demeester, "UGentWiLab, a real-life wireless sensor testbed with environment emulation", url: https://biblio.ugent.be/publication/676545 